\title{
Antioxidant capacity, phenolics and isoflavones in soybean by-products.
}

\begin{abstract}
This study aimed to determine proximate composition, antioxidant capacity, total phenolic content, isoflavones and free phenolic compounds in soy by-products. High carbohydrate and protein contents were found in grade A soymilk powder (GASP) compared to grade B soymilk powder (GBSP) and soy husk powder (SHP). Ash, moisture and total dietary fibre contents were reported to be the highest in soy husk, while GBSP had the highest fat content. Antioxidant capacity as assessed using $\beta$-carotene bleaching assay was in the order of SHP $\approx$ GBSP > GASP, and the ranking order of the Trolox Equivalent Antioxidant Capacity (TEAC) value was GASP $\approx$ GBSP $>$ SHP, while the Ferric Reducing Antioxidant Power (FRAP) value was GASP > GBSP > SHP. The total phenolic content was in the range of 62.44-103.86. mg GAE/100. g wet weight, and the major phenolic compounds in free form were ferulic, vanilic as well as gallic acids. Acid hydrolysis increased the amount of total extractable isoflavone in all soy samples.
\end{abstract}

Keyword: Antioxidant capacity; Isoflavones; Proximate composition; Soy by-products 